Part 1 of this paper (Harries and Harries 2001) examined the reasoning studies of the 1980s and 1990s and critiqued the ethnographic and informationprocessing approaches, based on stated information use. The need for an approach that acknowledged the intuitive nature of experienced thinkers' reasoning was identified.

Part 2 describes such an approach - social judgement theory - and presents a pilot application in occupational therapy research. The method used is judgement analysis. The issue under study is that of prioritisation policies in community mental health work. The results present the prioritisation policies of four occupational therapists in relation to managing community mental health referrals.

\title{
Studying Clinical Reasoning, Part 2: Applying Social Judgement Theory
}

\author{
Priscilla A Harries and Clare Harries
}

\section{Social judgement theory}

Social judgement theory offers a very different perspective to that of the information-processing approach (Doherty and Kurz 1996). It has been heralded as well suited to the study of clinical reasoning (Wigton 1996) and some of the earliest studies using this method examined clinical judgement (Hammond 1955).

Social judgement theory developed from the ideas of Egon Brunswik (1903-1955), an Austrian-American psychologist (Doherty and Kurz 1996). Brunswik was concerned that research into cognition was mainly experimental and lacked 'ecological validity'. Brunswik identified the need to use representative design to present information similar to the real environment. The research needed to examine people's perception and judgement while taking into account the probabilistic (unpredictable) nature of the environment. Brunswik recognised the need to understand the range of individuals' judgements in a range of situations. He developed a 'lens model' as a representation of the relationship between a person (or other organism) and his or her environment. His use of correlation statistics allowed the information used in making decisions to be identified (Doherty and Kurz 1996).

The importance of the probabilistic structure of the environment can be seen in a study by Tape et al (1991). The accuracy of physicians' judgements about the probability of pneumonia in patients was higher for physicians in Nebraska than for those in Virginia or Illinois. Accuracy was measured through comparison with X-ray results. However, accuracy should be measured not only from the point of view of the decision maker but also in the context of the predictability of the environment. When the environment was studied, it was found that the X-ray results had a clearer relationship with the symptoms in Nebraska than those in Virginia and Illinois. The physicians' use of information in making their judgement was appropriate for the environment (the set of cases) in which they practised.

\section{Rationale}

Judgement analysis, the methodology of social judgement theory, has significant potential for overcoming the limitations of a pure information-processing approach (Cooksey 1996). It has been successfully used in a wide variety of fields, such as medicine (Wigton 1988, 1996), finance (Waller 1988) and weather forecasting (Stewart 1990). It has also been successfully used to analyse the relationship between individuals' decision making in multidisciplinary rehabilitation teams that have included occupational therapists (for example, Unsworth et al 1997).

One rationale for using the methodology relates partly to the benefits that it has over an information-processing approach, which relies on the participant's ability to make policies explicit. Owing to the minimal processing and unconscious nature of some expert thinking, such an approach cannot be used to access the full range of cognitive processes. Judgement analysis only requires the decision maker to make judgements or decisions as he or she normally would. There is no requirement for the participant to access the processing stage. Judgement analysis does not, therefore, rely on insight, subjective interpretations, the ability to make implicit policy explicit or the ability to describe a process that has become minimised if not completely automatic and unconscious. Judgement analysis presents a statistical analysis describing the relationship between the information available and the judgement or decisions made.

The methodology involves presenting participants with a large number of computer-generated scenarios based on the types of information and the presentation of cases that would occur in the natural environment. Brunswik advocated the use of representative design (Doherty and Kurz 1996). However, often in judgement analysis, although any one judgement is made on a realistic scenario, the correlations between the types of information presented in the scenarios are minimised so that the role of any one piece 
of information can be clearly measured (orthogonal design) (for example, Evans et al 1995). This analysis can produce a prediction of policy use.

Although qualitative research wishes to gain insights of depth rather than breadth, quantitative research is necessary to access the wider view. An ethnographic approach may have advantages over judgement analysis in terms of the representativeness of the design, but it is difficult to observe a sufficient range of scenarios to generalise about how decisions are made. Interviews may give an opportunity for global issues of decision making to be discussed, but these are poor predictors of actual practice (Kirwan et al 1986) (see part 1, Harries and Harries 2001). It is difficult to discuss every possible scenario and even if the participants can describe their policy on any one scenario they may not be consistent in applying it. In the traditional methods for studying clinical reasoning by occupational therapists, a few carefully selected clinical scenarios are observed, described or generated. Therefore, statistical analysis cannot be conducted. No prediction of policy use for an individual or a group of individuals can be made.

Another benefit of using the judgement analysis approach is that the statistically identified policies can be compared with participants' subjective policies (how they think they have made their judgements) (Evans et al 1995). These can then be used to improve the practitioners' awareness of their practice policies.

\section{The pilot study}

With regard to this pilot study, the field of concern chosen was the reasoning used in the examination of referral data in community mental health. Previously, research in this field had been based on think-aloud techniques and interviews and only limited findings had been obtained (for example, Harries 1998). It was therefore felt appropriate to pursue the same field in order to see more readily the benefits of the judgement analysis approach. The issue of referral prioritisation was chosen as the specific decision under investigation for this judgement analysis study. The prioritisation policies of individual occupational therapists as opposed to community team prioritisation policies were of interest. Team prioritisation policies had already received research attention (Job 1996, Slade et al 2000) and the only study to have examined the policies used in the prioritisation of referrals sent directly to occupational therapists had used the information-processing approach (Harries 1998).

For many occupational therapists, team referrals are considered by a group of team members and make up the majority of referrals. However it is also common for occupational therapists to receive referrals directly from psychiatrists, general practitioners and fellow community mental health team members. An awareness of the occupational therapists' policies would benefit novice occupational therapists' understanding of the prioritisation issues when taking up a community post. Experienced occupational therapists' policies are necessary for training novice practitioners in undergraduate education in order to equip novice clinicians with the knowledge needed to manage their own caseload (Brunel University 1999). Policies derived previously have been of limited validity and reliability (Harries and Harries 1998). The aim of the pilot study, therefore, was to use judgement analysis to identify the policies that expert occupational therapists used in the prioritisation of occupational therapy referrals within the field of community mental health.

\section{Method}

\section{Social judgement theory principles}

The social judgement theory approach involves the use of the principles of 'probabilism', 'correlation statistics' and 'representative design' (Doherty and Kurz 1996).

\section{Probabilism}

'Probabilism' is the principle that recognises that the world is probabilistic and, therefore, that phenomena are not always predictable in the way that they occur or behave. Decision tasks vary widely so a large number of decision scenarios need to be provided to ensure sufficient variety. For this pilot study, each occupational therapist was presented with 90 referral scenarios. (This is an idiographic design in that the behaviour of each participant is examined separately [Cooksey 1996].) As the decision-maker's judgements may also vary even though the scenario may be the same, 30 repeats were added to examine the degree of consistency (intra-rater reliability).

\section{Correlation statistics}

'Correlation statistics' are used to compare the fit of the predicted policy model with its actual use, the degree of consistency of the decision-maker and the degree of agreement between decision-makers (inter-rater reliability). The effect of each cue is also measured in terms of how it varies as the judgement varies. As it is necessary to be able to see the effect of each cue independently, the correlations between the cues are minimised. This was done across all the referral scenarios $(r<0.02, n=90)$.

\section{Representative design}

'Representative design' refers to the realism of the scenarios. The scenarios used here were not fully representative because the intercue correlations did not match those in real life. Representative design could have been maximised by using large numbers of real referrals, sampled naturally and from a range of geographical areas. The statistics for analysing policy when cues hold their natural intercue correlations are more complex and the interpretability of analysis is compromised to a degree. However, this approach would be worth considering for future studies if such complex statistical support was available.

Both the content and the face validity were maximised. The content of the scenarios was based both on the results of previous research (Harries 1998) and on consultations with current practitioners. Harries (1998) elicited the content of the possible factors that are thought about as the 
community occupational therapist examines a referral. Thus, for example, the possible referrers and the type of diagnosis a client may have were identified. In addition, currently practising clinicians in the field were consulted to ensure that the factors were appropriate.

The presentation of the referrals was based on real referral forms in order to maximise face validity. One issue of concern related to the fact that here decisions could be based only on information in the referral forms. In the clinical setting, occupational therapists commonly see clients before making a final decision on referral prioritisation (Job 1996). On discussion with experienced clinicians in the field, it became apparent that the main reason for seeing clients was to validate the referral information. For example, was the reason for the referral the actual reason that the client needed to be seen? Whether the information came from a referral form or a first contact, it was the issue itself that gave the occupational therapist an indication of the level of prioritisation of need and, less importantly, the source of that information. The referral information was therefore felt to be a possible starting point for establishing prioritisation policies.

\section{Design of referrals}

In designing the referrals for the research, some factors were fixed in the referral whilst others were varied. Factors needed to be fixed if they were already known to have a categorical effect on the decision making. For example, the addresses of the clients had to be located within the catchment area of the team. If the clients lived outside the geographical area then the referral would always be rejected.

The varied factors (cues), which had the potential to influence the degree of priority given, were randomised into the computer-generated referrals using Visual Basic as the programming tool (carried out by $\mathrm{CH}$ ). These are shown in Table 1.

In order to give these variables numerical status for entering them into the statistical analysis, the content of the variables was rank ordered by a separate group of clinicians who did not participate in the study (details of the rank ordering are available from $\mathrm{PAH}$ ).

Each referral, generated on the computer, was presented on an individual piece of paper as a completed referral form (see Fig. 1). The participants were given their own bound book of referrals. All the participants saw the same set of referrals in the same order. They were allowed to make notes on the referrals and were able to move back and forward between the sheets. They were asked to make their own initial prioritisation ratings. For the purposes of later identification, very small numbers were written lightly in pencil on the back of the referral letters. The repeated referrals were given different numbers on their second presentation so that they could not be obviously matched.

\section{Participants}

Five occupational therapists were asked to participate in the pilot stage of this study. As the policies of experienced clinicians were wanted, only senior-level occupational
Table 1. Cues and their levels (rank orders not shown)

\begin{tabular}{|c|c|}
\hline Sex & Female \\
\hline & Male \\
\hline Age & 20-55 years old \\
\hline Referrer & General practitioner \\
\hline & $\begin{array}{l}\text { Colleague (community psychiatric nurse or } \\
\text { social worker) }\end{array}$ \\
\hline & Psychiatrist \\
\hline Diagnosis & Anxiety and depression \\
\hline & Obsessive compulsive neurosis \\
\hline & Depression \\
\hline & Schizophrenia \\
\hline & Anxiety \\
\hline Length of history & One-year history \\
\hline of mental health & Ten-year history \\
\hline problems & Five-year history \\
\hline Current living & Group home, staff live out \\
\hline situation & Home with family \\
\hline & Home alone \\
\hline $\begin{array}{l}\text { Reason for } \\
\text { referral }\end{array}$ & $\begin{array}{l}\text { Needs support, especially as embarking on a college } \\
\text { course. }\end{array}$ \\
\hline & $\begin{array}{l}\text { Managing work and maintaining friendships but } \\
\text { having difficulty getting on with family. }\end{array}$ \\
\hline & $\begin{array}{l}\text { Managed to stabilise drinking (3-4 pints per day); } \\
\text { persisting memory problems and quality of life issues. }\end{array}$ \\
\hline & $\begin{array}{l}\text { This client is not using their time very effectively but } \\
\text { lacks motivation to change. }\end{array}$ \\
\hline & Likely to relapse following imminent redundancy. \\
\hline & $\begin{array}{l}\text { Recent change in medication. Please support and } \\
\text { monitor in the community. }\end{array}$ \\
\hline & $\begin{array}{l}\text { Psychological and physical disabilities. Functional } \\
\text { assessment needed to identify level of support } \\
\text { required. }\end{array}$ \\
\hline & $\begin{array}{l}\text { Lost confidence with going out and is not looking } \\
\text { after themselves very well. }\end{array}$ \\
\hline Other services & Day centre \\
\hline involved & Counsellor \\
\hline & No other \\
\hline Any known & No \\
\hline history of & Verbally abusive \\
\hline violence & Physically abusive \\
\hline & Suicidal \\
\hline
\end{tabular}

therapists were recruited. To ensure that the occupational therapists were those with well-developed prioritisation policies, only clinicians who had been in their current post for a minimum of 6 months were recruited. Confidentiality was assured for all participants. They were allocated a code prior to data collection and the names and codes were held separately.

\section{Procedure}

The participants completed three tasks. The first task required them to indicate the priority of each of the 120 


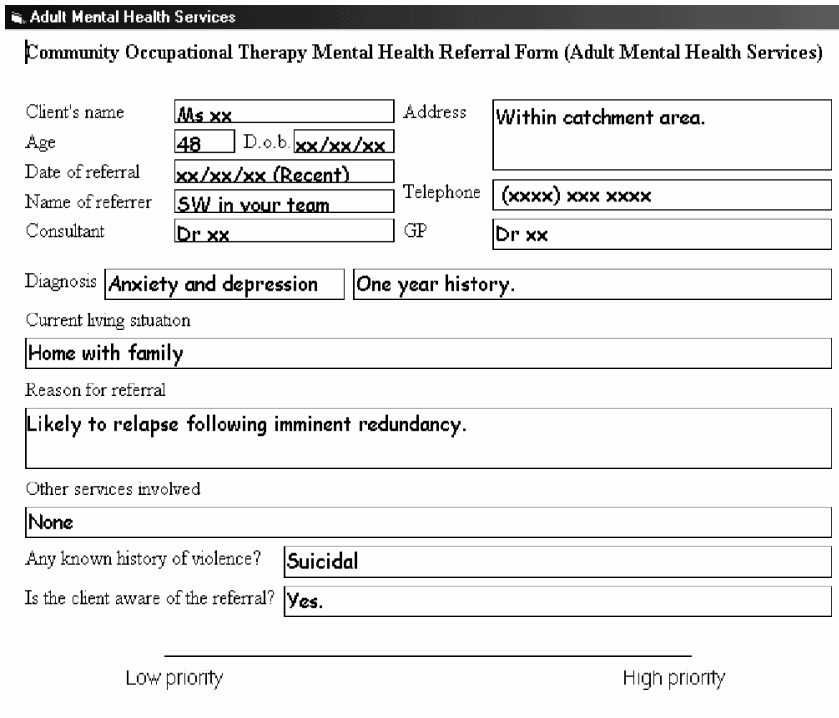

referrals by marking a cross along a visual analogue scale, labelled 'low priority' at one end and 'high priority' at the other (see Fig. 1).

The second task asked them to state the policies that they thought they had used over those referrals. This involved both rating each of the possible pieces of referral information in relation to the influence it had in terms of prioritisation and indicating how each level of the variable related to the prioritisation judgement, independently of other factors. Subjective ratings were between 0 and 10, with 0 indicating no bearing on prioritisation of referral and 10 indicating high bearing on prioritisation of referral. The participants could use any number more than once. The effect of each level of the variables was indicated using several visual analogue scales.

The third task was to complete a questionnaire based on demographic data. It was expected that the use of different factors in prioritisation judgements might be related to practice settings and the previous experience of the individual participants. All three tasks were posted to the participants, to be completed individually and then returned to the researcher.

The final procedure of the study, which took place after data analysis, involved providing the participants with feedback on their subjective and objective policies. They then had an opportunity to comment on the findings.

\section{Types of analys is}

There were four types of analysis.

\section{Analysis of tacit judgement policies}

Following the standard practice of judgement analysis, the prioritisation policies were obtained using multiple regression analysis (Cooksey 1996). This provides the weighting of the individual variables, such as the bearing that the client's diagnosis had or the bearing that the reason for referral had. The fit of the policy $(\mathrm{R})$, described by the multiple linear regression, was calculated: judgements were predicted from it and these were correlated with the actual judgements made.
Multiple linear regression assumes a linear relationship between the variable and the prioritisation judgement. This may not have been the case with the rank ordering in the study variables. To examine this, the relationship between each variable and the judgement was plotted. For example, a significant weighting of diagnosis could have been reflective of the importance of schizophrenia over the other diagnoses, rather than a steady increase of importance with different diagnoses.

\section{Comparison with stated judgement policies (self-insight)}

Self-insight was examined by comparing the statistical analysis of information use with how participants stated that they had used the information.

\section{Consistency and agreement}

The intra-rater reliability, that is how consistent an individual was in his or her own policy use, and the interrater reliability, that is how much agreement there was between participants, were both identified using the Pearson's correlation test ( $r$ ). To measure consistency, the correlation compared the prioritisation ratings on the 30 repeat referrals with the prioritisation ratings made on the same 30 referrals in the main set. To measure agreement, the correlation compared the set of prioritisation ratings given by each participant on the main set of 90 referrals.

\section{Results}

Four of the five occupational therapists returned the data packs.

\section{Analysis of tacit judgement policies}

A tacit judgement policy was calculated for each of the four participants. Tacit judgement policies consisted of sets of standardised regression coefficients. These are a measure of how much the judgement varies as the cue varies. The larger the regression weight, the larger the impact of the cue. The size of these standardised regression coefficients (the blocked-in bars) are shown for each participant in Fig. 2. The $\mathrm{x}$-axis shows the nine different cues (independent variables). Cues are usually defined as being used if their regression coefficient is significantly different from zero $(p<0.05)$. These are identified with an asterisk in Fig. 2

Participant 1 used the client's living situation and the client's history of violence. Participant 2 used the diagnosis and the reason for referral. Participant 3 used the client's history of violence and the level of support that the client had. Participant 5 used the client's living situation, the client's history of violence and the level of support that the client had. The fit of each regression model was calculated by taking the predicted judgements (standardised weights $\mathrm{x}$ value within standardised range) and correlating them with the actual judgements over the 90 cases. The scores for the fit of the model, R, were $0.68,0.72,0.77$ and 0.65 for the four respective pilot participants. (Perfect fit is $R=1$.) The fit was therefore reasonably good. 

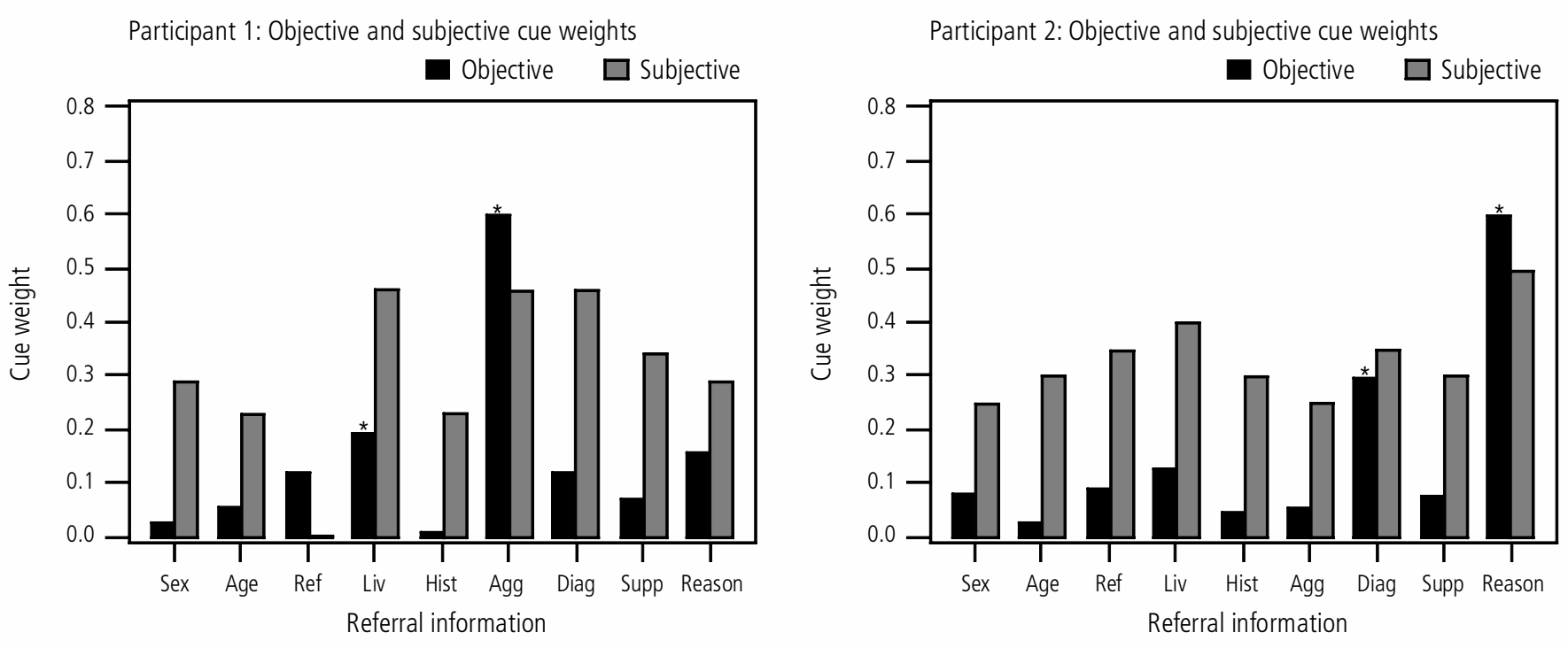

Participant 3: Objective and subjective cue weights

- Objective $\square$ Subjective

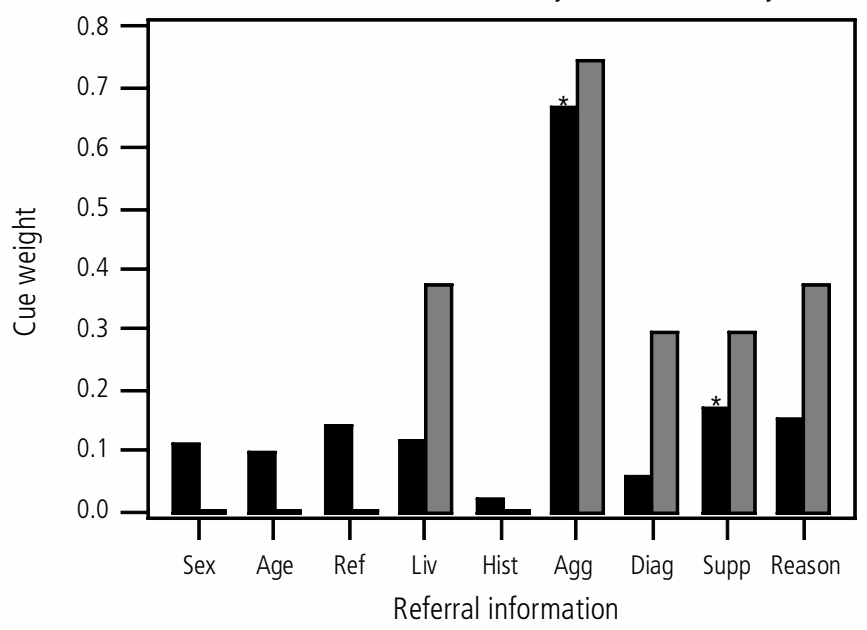

Participant 5: Objective and subjective cue weights

- Objective $\square$ Subjective

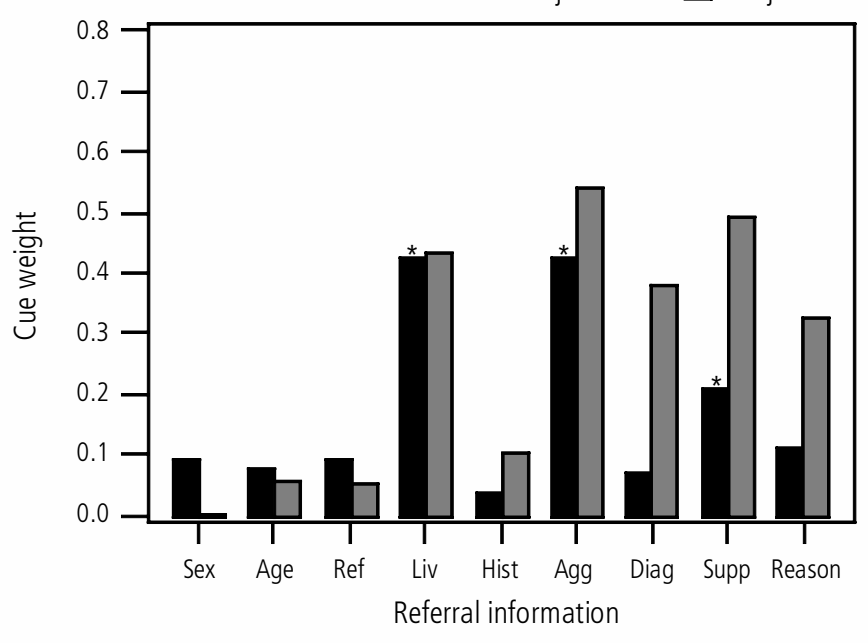

Ref = Referrer; Liv = Living situation; Hist = Length of history of mental health problems; Agg = Aggression (Any known history of violence); Diag $=$ Diagnosis; Supp $=$ Support (Other services involved). ${ }^{*} p<0.05$.

This global measure of use assumes a linear relationship between cues and judgements. For several variables it was one level of the variable, rather than an increasing trend, that led to changes in judgement.

The reason for referral was significant because the degree of occupational dysfunction was influential in determining the priority rating. Living situation was significant because those living alone, as opposed to those with family or in group homes, were highly prioritised. Available support was significant because those with no support as opposed to those with a day centre place were prioritised. Those who were suicidal (aggressive to self) or physically abusive were prioritised over those who were verbally abusive. Those with schizophrenia were prioritised over those with other diagnoses such as neurotic disorders.

\section{Comparison with stated judgement policies (self-insight)}

The four graphs in Fig. 2 also provide a global comparison between the actual and the stated policies. This is a measure of the participants' level of self-insight. The subjective rating of each variable was standardised by dividing it by the standard deviation of predictions of prioritisation judgements over all cases made from these subjective ratings (a method designed by Ian Dennis; see Harries [1995, p269] for methodology). These are shown as shaded bars in Fig. 2 and represent the participants' subjective policies. It was found that all four participants had some degree of insight into the factors that were of key importance, but usually overestimated the number and degree of importance of most of the other cues.

In addition, a more specific analysis of participants' selfinsight as to the importance of the role of different levels of a cue was carried out. For example, if the participants had used diagnosis as an influential cue, were they aware of the influence of the different types of diagnosis? The subjective importance of each level of a cue was elicited from the participants by asking them to mark off, on a line, the average prioritisation given to people with that level of that cue, all other things being equal. It was found that for those cues that had actually been used in the prioritisation task, 
the participants showed good self-insight. They were able to identify the order in which the cue level had influenced the degree of prioritisation. However, because they had overestimated the number and degree of cues that had not been used in the actual task, they also identified the cue levels as more influential than they actually were. Some cue levels, which had not actually been used at all, were usually thought to be used at or around the same degree of influence as the cue levels that had actually been used.

\section{Consistency}

The participants' ability to apply their own policies consistently was found to vary greatly. This was established by comparing the scores of the 30 original and the 30 repeated referrals. The most consistent policy was where $r=$ 0.87 . The least consistent policy was where $r=0.3$. (A perfect match is seen when $r=1$.)

\section{Agreement}

Inter-rater reliability also varied greatly. This was measured by comparing pairs of participants' prioritisation ratings across all 90 cases. The poorest match was between participant 1 and participant 2, where the Pearson's test was 0.226 ( 1 being a perfect match). The best match was between participant 1 and participant 3 , where the result was 0.68 .

\section{Discussion}

\section{Prioritisation policies}

The participants' policies for prioritising referrals could be identified using the methodology of judgement analysis. A discussion of the statistically significant factors used in the prioritisation decisions is necessary to understand their relevance to the field of study. The regression models were reasonable fits for the prioritisation judgements of each participant. Policies described therefore have potential as a basis for the education of novice practitioners.

\section{Degree of occupational dysfunction}

The greater the degree of occupational difficulties contained in the reason for referral, the higher was the priority given. This may seem an obvious indicator for an occupational therapist's involvement. However, only one participant used it as a statistically significant factor. By examining the demographic data and discussing the finding with the participants, it became apparent that the degree to which this cue was an influential factor was related to the nature of the therapist's role. The participant who took a minimal generic role and a mostly profession-specific role had been the only one to use this factor significantly. The participants that had not been as focused on this factor (factor of borderline significance, $\mathrm{p}<0.07$ ) were taking a generic role with the majority of their clients. Therefore, the participant wishing to keep a more profession-specific role to work was found to be more likely to take account of the level of occupational dysfunction in the reason for referral.

\section{Living alone, no support}

These issues could result in an isolated client trying to function independently. They would have an effect on the client's ability to function and would therefore draw the attention of the occupational therapist.

\section{Suicide risk}

Targeting those with a suicide risk is recognised as being part of a larger governmental prioritisation policy (Department of Health 1995). Community mental health teams would have an important role in helping to achieve this and are encouraged to support this policy. Clients who are physically abusive pose a threat to the community, an issue that is of concern to the community and professionals in general.

\section{Schizophrenia}

According to government policy (Department of Health 1995), clients with severe enduring mental health needs such as chronic schizophrenia are also to be prioritised by community mental health teams. Schizophrenia is recognised as having a more detrimental effect on occupational functioning than other diagnoses, such as anxiety and depression, and would draw the particular attention of the occupational therapist. It was interesting that the length of history was not used as a significant factor when this is also part of the government policy (Department of Health 1995). During the feedback of results to the participants, this was raised. The participants acknowledged that if a client had a short history they would be keen to prioritise him or her. Their reasoning related to their wish to address a client's needs at an early stage in order to prevent any long-term problems.

\section{Self-insight}

All four participants had some self-insight into the factors that were of key importance. They also had good insight into the importance of the levels of these cues. This degree of accuracy was impressive and showed a level of self-insight not always found in other studies of clinical policy awareness (Shanteau 1992). Although aware of the main cues used, the participants had, for almost all the other cues, overestimated their degree of relevance. The overestimation of the number and relevance of cue use is a finding that is commonly noted in other studies. There are a number of different possible reasons for this (Harries and Harvey 2000, Harries et al 2000).

What is perhaps important to note is that this mixed picture of self-insight, that is some accuracy and some overestimation, may account for some of the difficulties in gaining an accurate picture of policy use when using the information-processing approach. Insight into the key factors would allow the influential factors to be presented, but these would be discussed alongside a lack of insight into the many non-influential factors. This clouding of the issues of importance would make it hard to gain an accurate impression of policy use. The accuracy of accessing the 
policy would also, as discussed in part 1 , be compounded by the difficulty in separating out the policy that is being applied to the decision under study as opposed to policies being applied to other concurrent decision processes, such as the treatment and management decisions. The judgement analysis approach has the benefit of not relying on accessing the processing of the decision and, therefore, is not dependent on insight into established policy use.

\section{Consistency and agreement}

Both the clinicians' ability to apply their own policies consistently and agreement on their policy use were found to vary greatly. Applying policies as a professional group has been found to be generally poor in clinical studies. This has been attributed to the lack of feedback that clinicians receive after making their judgements (Shanteau 1992). It can be seen to be in contrast to weather forecasters, who receive feedback when the weather arrives and have therefore been able to become fairly consistent in policy use both as individuals and as a group (Shanteau 1992). Clinicians ultimately alter the needs of clients if they come into contact with them and it is difficult, therefore, to receive feedback on what would have happened to the clients had they not been seen.

\section{Critique of methodology}

Some of the pilot study participants reported that they had found the task of prioritising 120 referrals very demanding cognitively. The size of the task was necessary to allow essential statistical analysis to be conducted. In order to keep the demands at an acceptable level, judgement analysts recommend that rest breaks be taken if the total number of profiles exceeds 100 (Cooksey 1996). In the letter of instruction, the participants were encouraged to take breaks when required. As in the clinical setting, referrals would be viewed in batches.

Additional referrals could have been added to the set to allow for 'judgemental bootstrapping' (Cooksey 1996), a method of checking if the captured policies would be reliable predictions of participants' policies. However, these were not added to the set of 120 referrals because it appeared that the participants' maximum capacity for the task had already been reached.

Another issue of concern raised by the participants was that the range of variables within each cue was less varied than those experienced clinically. For example, only five diagnoses were used in the referrals whereas 10 or more may be experienced in the clinical setting. Had a wider range of the variables been included in the referrals, the total number of referrals would have had to be increased. As has just been discussed, this would have caused the total task to have become cognitively unmanageable. It is also worth remembering that the main purpose of the research was to identify policies for education purposes. Undergraduate students would be required to learn the prioritisation policies of only core referral data rather than those of all possible scenarios.

\section{Conclusion}

Judgement analysis provides a methodology that can identify how information has been used and combined in decisions. The results account for the full spectrum of reasoning, from the analytical to the intuitive. This is especially important in allowing the understanding of the valuable clinical policies of experts, particularly when intuitive decision making has occurred. This method, as long as the tasks are represented accurately, will allow occupational therapists to tap reliably a previously unavailable source of knowledge.

The policies of four experienced occupational therapists have been captured at this pilot phase. The techniques of data collection and analysis will require refinement. This is especially important in order to maximise the accuracy of collecting the subjective policies. However, the subjective policy data remain dependent on information-processing methods for collection, which are recognised as having limitations (Doherty and Kurz 1996). The use of multiple linear regression assumes a linear approach in the use of information. In the study, this analysis was complemented with a specific examination of the effects of different levels of each cue. In addition, the participants were allowed to indicate the effect of the different levels of the cues as well as rating the overall influence of the cue on their judgement making. Further analysis along these lines would be useful.

The next stage of the research will involve collecting policies from about 40 participants in order to establish trends in prioritisation policy use. This information may be of interest to practising occupational therapists, not only as a means of improving their self-insight in terms of noninfluential factors but also to develop their awareness of others' practices. The prioritisation policies and the settings in which they may be appropriately applied can then be used in education. Methods for teaching these policies, such as training with outcome feedback or cognitive feedback (Balzer et al 1989, Wigton et al 1990, Tape et al 1992), need to be experimentally tested to understand which methods will be the most effective for the education of prioritisation policies.

\section{Acknowledgements}

Thanks to the four clinicians who participated in the pilot study. Thanks also to Professor Ken Gilhooly for his advice and support.

\section{References}

Balzer WK, Doherty ME, O'Connor R (1989) Effects of cognitive feedback

on performance. Psychological Bulletin, 106, 410-33.

Brunel University (1999) BSC (Hons) Occupational Therapy: student course handbook. London: Brunel University.

Cooksey RW (1996) Judgement analysis: theory, methoos and

applications. London: Academic Press.

Department of Health (1995) Building bridges: a guide to inter-agency working for the care and protection of severely mentally ill people. London: HMSO.

Doherty ME, Kurz EM (1996) Social judgement theory. Thinking and

Reasoning, 2, 109-40.

Evans JS, Harries C, Dennis I, Dean J (1995) General practitioners' tacit and 
stated policies in the prescription of lipid lowering agents [see comments]. British Journal of General Practice, 45, 15-18.

Hammond KR (1955) Probabilistic functionalism and the clinical method.

Psychological Review, 62, 255-62.

Harries C (1995) Judgement analysis of patient management: general practitioners' policies and self-insight. Unpublished PhD thesis. Plymouth: Psychology Department, University of Plymouth.

Harries C, Evans JSBT, Dennis I (2000) Measuring doctors' self-insight into their treatment decisions. Applied Cognitive Psychology, 14, 455-57.

Harries C, Harvey N (2000) Taking advice, using information and knowing what you are doing. Acta Psychologica, 104, 399-416.

Harries PA (1998) A study to identify, in the field of community mental health, the factors influencing occupational therapists' decision making as to whether or not to accept a referral (Abstract). British Journal of Occupational Therapy, 61, 156.

Harries PA, Harries C (1998) Listening to reason: accounting for clinical decisions. Book of Abstracts, 12th Intemational Congress of the World Federation of Occupational Therapists. Montreal: WFOT.

Harries PA, Harries C (2001) Studying clinical reasoning, part 1: have we been taking the wrong 'track'? British Journal of Occupational Therapy, 64(4), 164-68.

Job T (1996) A system for determining the priority of referrals within a multidisciplinary community mental health team. British Joumal of Occupational Therapy, 62, 486-90.

Kirwan JR, Chaput de Saintonge DM, Joyce CR, Holmes J, Currey HL

(1986) Inability of rheumatologists to describe their true policies for assessing rheumatoid arthritis. Annals of Rheumatic Disease, 45, 156-61.

Shanteau J (1992) Competence in experts: the role of task characteristics. Organizational Behavior and Human Decision Processes, 53, 252-66.

Slade M, Powell R, Rosen A, Strathdee G (2000) Threshold assessment grid (TAG): the development of a valid and brief scale to assess the severity of mental illness. Society of Psychiatry and Psychiatric Epidemiology, 35, 78-85.
Stewart TR (1990) A decomposition of the correlation coefficient and its use in analysing forecasting skill. Weather and Forecasting, 5, 661-66.

Tape T, Heckerling P, Ornato J, Wigton R (1991) Use of clinical judgement analysis to explain regional variations in physicians' accuracies in diagnosing pneumonia. Medical Decision Making, 11, 189-97.

Tape TG, Kripal J, Wigton RS (1992) Comparing methods of learning clinical prediction from case simulations. Medical Decision Making, 12, 213-21.

Unsworth CA, Thomas SA, Greenwood KM (1997) Decision polarisation among rehabilitation team recommendations concerning discharge housing for stroke patients. International Journal of Rehabilitation Research, 20(1), 51-68.

Waller WS (1988) Brunswikian research in accounting and auditing. In: B Brehmer, CRB Joyce, eds. Human judgement: the SJT view. Amsterdam: North-Holland, Elsevier Science.

Wigton RS (1988) Applications of judgement analysis and cognitive feedback to medicine. In: B Brehmer, CRB Joyce, eds. Human judgement: the SJT view. Amsterdam: North-Holland, Elsevier Science. Wigton RS (1996) Social judgement theory and medical judgement. Thinking and Reasoning, 2, 175-90.

Wigton RS, Poses RM, Collins M, Cebul RD (1990) Teaching old dogs new tricks: using cognitive feedback to improve physicians' diagnostic judgements on simulated cases. Academic Medicine, 65(9), (Suppl), S5-S6.

\section{Authors}

Priscilla A Harries, DipCOT, MSc, SROT, Lecturer, Department of Health Studies, Brunel University, Osterley Campus, Borough Road, Isleworth, Middlesex TW7 5DU.

Clare Harries, BA, PhD, Research Fellow, formerly at the Department of Psychology, University College London, and now based at the Centre for Decision Research, Leeds University Business School, Maurice Keyworth Building, University of Leeds, Leeds LS2 9JT.

\section{New N/SVQ Guidelines}

The College of Occupational Therapists encourages its members to carry out Continuing Professional Development and sees N/SVQ as part of this process of continuous updating, reflection and improvement of quality. A new document is now available for occupational therapist and their support staff to provide general information about N/SVQs.

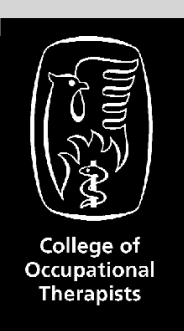

These guidelines will:

- help to clarify the N/SVQ process,

- give useful examples,

- answer frequently asked questions,

- and explain how to assemble a portfolio.

The document is available from: Publication orders, The College of Occupational Therapists, 106-114 Borough High Street, London SE1 1LB. Cost: $£ 3.00$ for BAOT Members, $£ 6.00$ for Non-Members. Please send a cheque made payable to the College of Occupational Therapists. 